\title{
An Introduction to Socially Connected Machines: Characteristics and Applications *
}

\author{
Taehyoung Shim, Dong Min Kim and Seong-Lyun Kim \\ Department of Electrical and Electronic Engineering, Yonsei University \\ 50 Yonsei-Ro, Seodaemun-Gu, Seoul 120-749, Korea \\ (e-mail: \{teishim,dmkim, slkim\}@ramo.yonsei.ac.kr)
}

\begin{abstract}
:
Due to the development of information and communication technologies, it is difficult to handle the billions of connected machines. In this paper, to cope with the problem, we introduce machine social networks, where they freely follow each other and share common interests with their neighbors. We classify characteristics and describe required functionalities of socially connected machines. We also illustrate two examples; a twit-bot and maze scenario.
\end{abstract}

Keywords: Machine-to-machine communications; machine social networks.

\section{INTRODUCTION}

Due to the development of information and communication technologies, the number of connections has been increased not only between people but also between machines [1]. According to an Erisson report, there will be 50 billion machines connected to the networks by the end of 2020 [2]. In current machine networks, however, it is difficult to handle the billions of connected machines [3]. To manage it more efficiently, it is needed to investigate how the machines discover the others and connect to one another for exchanging valuable information.

In social network services, we have seen that a large number of people can be neighbors who have the same interests. As gathering together in the social networks, people are more close, i.e. the average number of hops to the others has been decreased. Thus, they are easy to find their friends who have the same interests [4].

Through the intuition of social networks, some previous works changed the point of views on the machine networks. In $[3,5,6,7]$, the authors applied the social concept into the machines to solve the problem of network scalability. However, these works still stay showing a what-if scenario.

In this paper, we focus on introducing the characteristics of machine social networks (MSN), where socially connected machines (SCMs) freely follow each other and share the same interests with their followers. In the MSN, SCMs will mediate the interactions not only between people but also the SCMs, and they carry useful information to the others.

\footnotetext{
* "This research was supported by the MSIP(Ministry of Science, ICT and Future Planning), Korea, under the CPRC(Communications Policy Research Center) support program supervised by the KCA(Korea Communications Agency)" (KCA2013-001).
}

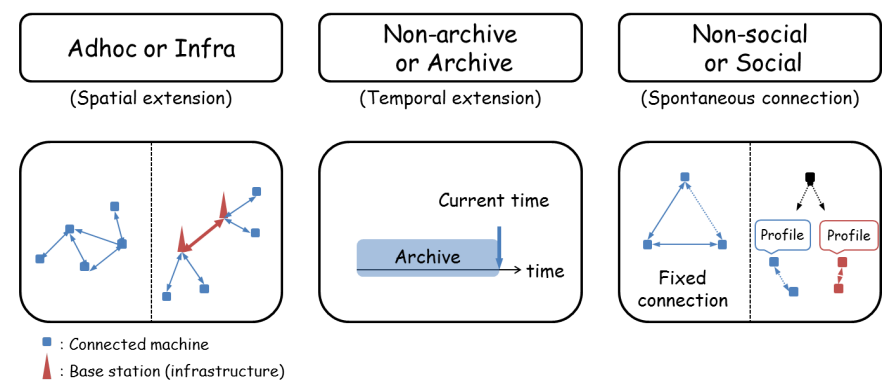

Fig. 1. A taxonomy of machine networks.

Throughout this paper, we introduce the characteristics of the SCMs, and then we suggest social connection elements that are composed of interest, space, and neighbor axis to assess the characteristics of the connections. By using the proposed properties, we describe some examples to illustrate the feasible scenarios in the MSN.

The rest of this paper is organized as follows: Section 2 classifies social machine networks. Section 3 describes the characteristics of the social machines. In Section 4, we show the examples that indicate feasible scenarios in MSN. Finally, Section 5 concludes the paper.

\section{MACHINE NETWORKS: TAXONOMY}

In this section, we classify machine networks into spatiality, durability, and sociality as shown in Figure 1. Thus, taxonomy of the machine networks can be explained as below.

\section{- Spatiality}

From a spatial perspective, we divide the machines into infrastructure and adhoc networks. If the machines use fixed networks (i.e., a wireless access point), they can access other machines over the limited space by using the Internet. This case is an infrastructure type network. On the other hand, in 


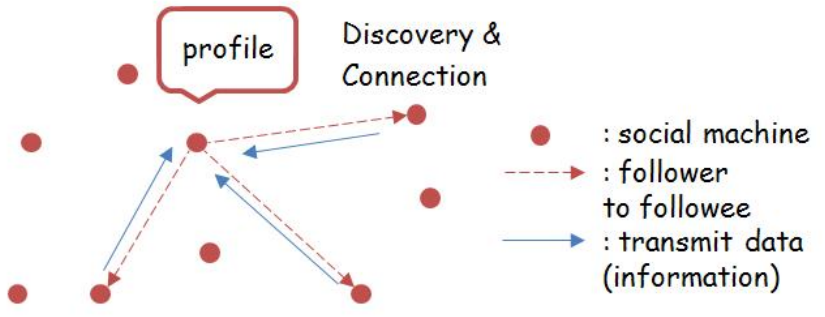

Fig. 2. Description of machine social networks.

the adhoc type network, the SCMs can only communicate with nearby neighbors, since the machines communicate via wireless links.

- Durability Depending on the possibility of accumulation of knowledge, we can define a durability in the machine networks. If the machines can freely use storages that are shared with the others, then this case is an archive type network. On the contrary, the network of the machines is a non-archive type if they can share only volatile information.

\section{- Sociality}

The machines share the information with the others by using the communication capability. Depending on the degree of a connection freedom, we divide the type of the machine networks into non-social network and social network. The machine network is non-social if the machine in the network is controlled by people or can share the information with only limited machines. The connection is fixed in the non-social network. On the other hand, if the connected machines freely follow each other and spontaneously share the information with their followers, the network is social.

According to the taxonomy of the machine networks, the machines belong to each of the classifications. The machines using social connection are called the socially connected machines (SCMs). In the next section, we describe the SCMs in detail.

\section{SOCIALLY CONNECTED MACHINES}

For achieving purposes, the SCMs connect the others with the same interests spontaneously to cooperate mutually. The SCMs have high connection diversity, public information and the ability to have multiple purposes. To realize the MSNs, SCMs should have following functionalities: announcing identification, discovering neighbors, having multiple interests and determining trustworthiness.

\subsection{Characteristics}

In Figure 2, we illustrate the structure of the MSNs. The machines who want to obtain information from their neighbors compose a network. The information provider is followee, and the receivers are followers. To discover the appropriate information provider, each machine investigates the profiles of the neighbors. By using their profiles, the SCMs receive the information from them. The characteristics of the SCMs are explained as below.

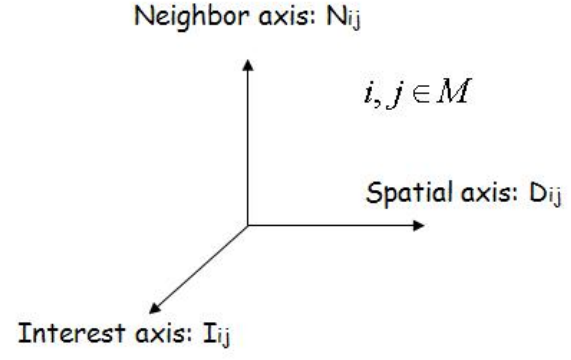

Fig. 3. Interest, spatial, and neighbor axis in machine social networks.

\section{- Connection diversity}

One of the characteristics is connection diversity. As mentioned in the previous section, the non-social machines have the fixed connection that only share the piece of information with limited machines. On the other hand, the SCMs have a variety of the connections depending on the interests. The possible number of connections between SCMs is greater than the number of connections in non-social network. As varying the space and time, the SCMs choose various machines to share the interesting information. Therefore, the diversity of the connections increases. In the MSN, the connections changes more frequently than non-social network.

\section{- Public information}

Each machine should open to the public the profile to allow accessing it. In MSNs, any SCM can freely access and widely disseminate the information to the others. Because of the lower privacy standards for the machines, the information propagation is relatively easy. The SCMs may be vulnerable from a spread of misinformation and a malicious attack.

\section{- Multiple purposes}

The SCMs have not only one-purpose but multipurpose. Since their interests vary in different space and time, they can serve multiple applications. As allowing multi-purpose to the machines, we reduce cost of machines efficiently.

\subsection{Required Functionalities}

To implement the SCMs, following functionalities are required.

\section{- Identification}

The SCMs need a machine profile that describes their identification information, such as operating time, location, interests, capabilities (e.g., kinds of sensors, peak velocity, amount of memory).

\section{- Discovery neighbors}

The SCMs should autonomously discover their neighbors who have the same interests.

\section{- Multiple interests}

The SCMs can have the multiple interests and a 


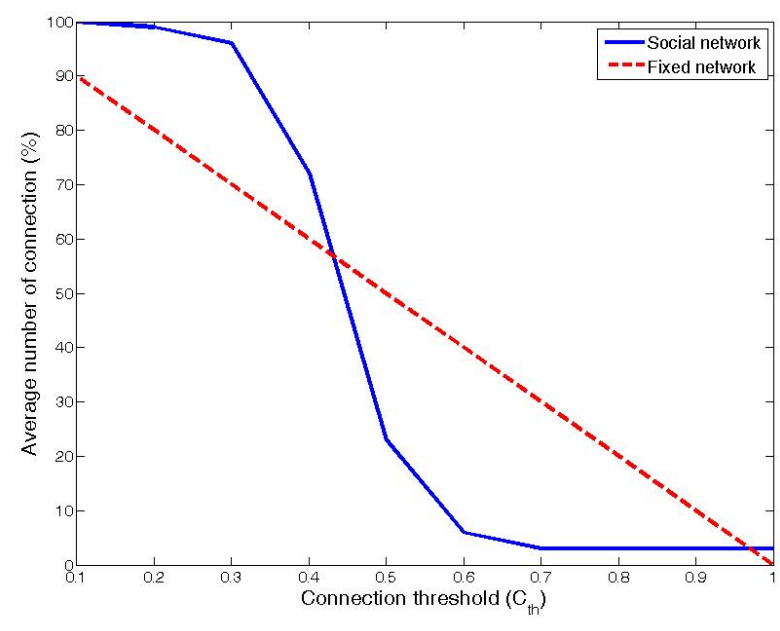

Fig. 4. Average number of connections.

capability of distributed tasks.

\section{- Trustworthiness}

The SCMs should have a capability that determines the reliability of other machines [3]. If the SCM connects with the malicious machine and is attacked, the SCM should exclude the spiteful machines from MSN.

\subsection{Connection between SCMs}

In this subsection, we explain that how the SCMs find their neighbors who have the same interests. To make possible the social network of machines, they have to discover the neighbors by using their profiles.

A relationship between machines $i$ and $j$ is established when the condition of the connected machines is met. Let $C_{i j}$ be the measure of the strength of the connection from $i$ to $j$. If the following condition is satisfied, the connection is established:

$$
C_{i j} \geq C_{t h}, i, j \in M,
$$

where $C_{t h}$ is the threshold to make a connection between machines, and $M$ denotes a set of the machines in machine networks. The threshold means a capability of machines in order to communicate each other. The social machine $i$ follows the machine $j$ if the relationship between $i$ and $j$ is bigger than the threshold. For discovering, we consider three elements: interest, spatial, neighbors factor between two machines as shown in Figure 3. We define the relation between the machine $i$ and $j$ as

$$
C_{i j}=w_{I} I_{i j}+w_{D} D_{i j}+w_{N} N_{i j},
$$

where weighted factors of the relationships are $w_{I}+w_{D}+$ $w_{N}=1$. In (2), first, the interest axis is $I_{i j}=\mid I(i) \cap$ $I(j)|/| I(i) \mid$, where $|I(i)|$ denotes the number of interests of the machine $i$. It means a correlation of interests between the machine $i$ and $j$ in the machine networks. If the machine $i$ and $j$ are working simultaneously with all of the same interests, a value of the correlation is equal to one. Second, the spatial axis, $D$ represents $D_{i j}=1-$ $\operatorname{dist}(x(i)-x(j)) /|x|$, where $x(i)$ means location of the

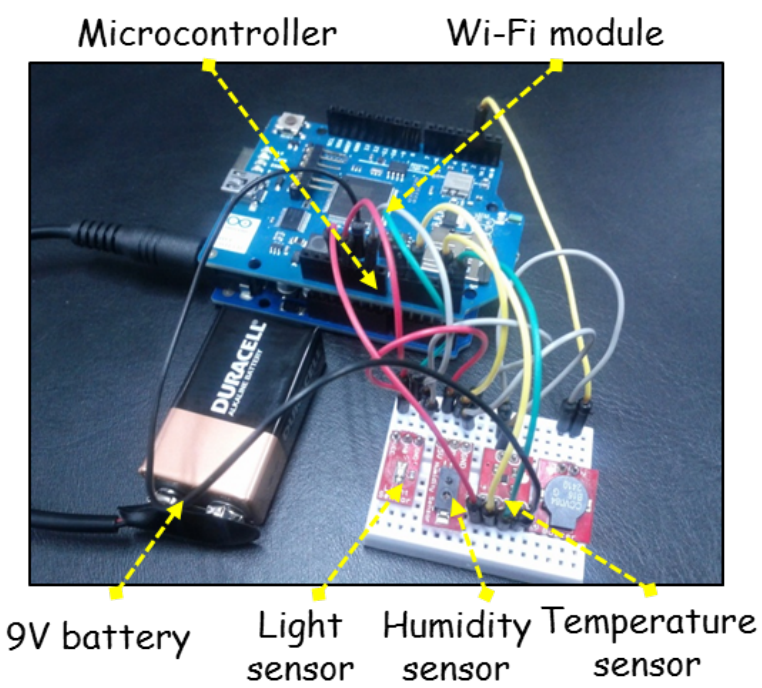

Fig. 5. A socially connected machine with sensors.

machine $i, \operatorname{dist}(\cdot)$ is the Euclidean distance. It means distance between two machines. The maximum distance between two machines is $|x|$. As close as possible, the spatial correlation between two machines $D_{i j}$ goes to one. Finally the social axis, $S$ means that how many they have mutual neighbors. The mutual neighbors is $N_{i j}=\mid N(i) \cap$ $N(j)|/| N(i) \mid$, where $N(i)$ denotes the set of neighbors of machine $i$. If two machines have a lot of mutual neighbors, then they make a neighbor relationship more easily.

As time goes by, the connected machines have the same level of information. It means the follower has no reason to follow the followee. We model this situation as follows:

$$
C_{i j}(\Delta t)=e^{-a \Delta t}<C_{t h}
$$

where $a$ is a constant value, and $\Delta t$ denotes an elapsed time from a time of making a neighbor. The strength of the social relation between two machines decays exponentially. In the MSNs, the social connection between two machines makes or deletes a link repeatedly.

\subsection{Social Connection Simulation}

To evaluate the performance of the SCMs, we simulate the MSNs and show that how the average number of connections changes with various connection thresholds. To compare to the performance, we use non-social networks with fixed connections.

We set the total number of machines is 100. In one dimensional space, there are 10 subspaces. Each machine exists one of subspaces. If there are two machines in the same subspace, then the distance is zero and $D_{i j}$ is one. The total number of interests is ten, and each machine has five interests. In this network, the SCMs make neighbors and disconnect with them repeatedly. As we mentioned the threshold $C_{t h}$, it means a capability of machines in order for communicating each other. If it goes to one, then making the connection between the machines is very low. If the threshold is extremely high, the machines can not make a group and be separated in the network. 


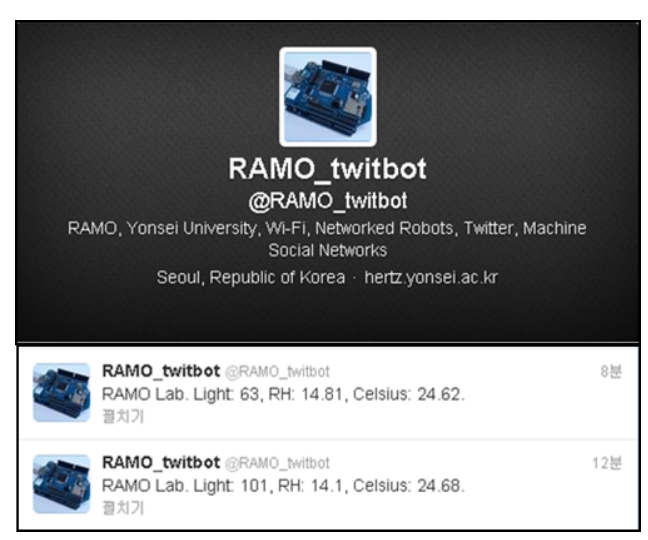

Fig. 6. Social network platform: Twitter.

Simulation result In Figure 4, we evaluated the average number of connection according to the connection threshold $C_{t h}$. The connection threshold is a technology that is correlated with discovering other machines and transmitting capability. If the connection threshold is low, then a dissemination of information is high. As shown in Figure 4, the SCMs (solid line) is isolated if the connection threshold is low. It is shown that the number of connection between the machines is higher than typical fixed machine networks (dotted line) if the connection threshold is lower than 0.45. This means that the SCMs can make a connection or a group if the machines have lower threshold. This simulation result shows that the connection threshold should be relatively low in order to take advantage of the MSN.

\section{MACHINE SOCIAL NETWORK APPLICATIONS}

In this section, we suggest two examples in MSN. One is a twit robot, and the other is a maze scenario. According to the machine taxonomy, the twit bot has properties; infrastructure, archive, and social. Another example has properties; Adhoc, Non-archive or Archive, and Social.

\subsection{Twitbot}

The SCMs transmit the information to the followers automatically. They are able to discover neighbors who have the same interests and connect with them.

As shown in Figure 5, we installed one example of the SCMs. Our machine is composed of a micro controller, a wireless module, and several sensors. We use arduino uno board that is easy to be controlled a micro controller by using c language. In order to connect to the Internet conveniently, we adopted arduino wi-fi shield that conforms to standard IEEE $802.11 \mathrm{~b} / \mathrm{g}$ [8]. Our machine also has sensors; light, humidity, and temperature sensors. From these sensors, we could sense our environments.

This machine periodically perceived changes in surrounding environment, for example, notifying weather changes, and posting critical information on its web page, such as Twitter. Followers of this social machine can receive the information periodically or in emergency situations. Our SCM periodically sends the information to its followers. In order to make a social relationship with others, it should discover others who have the same interests.

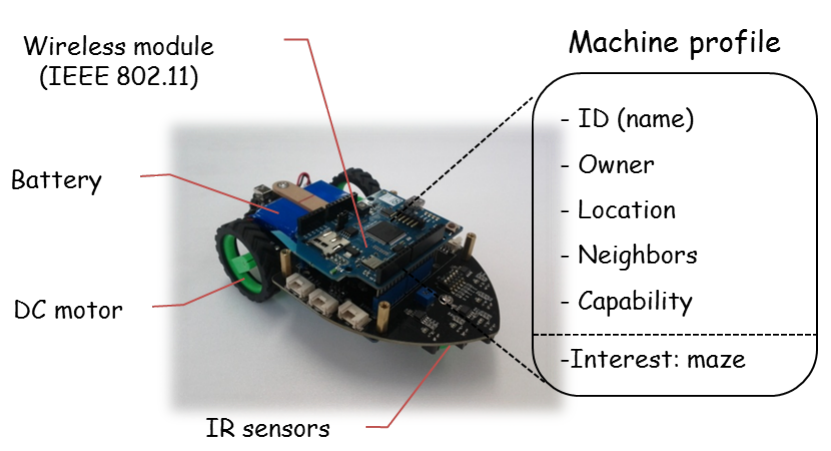

Fig. 7. Socially connected machine in a maze.

\subsection{Socially Connected Machines in Maze}

In [9], the authors referred that machines can cooperatively solve a maze problem by using wireless multihop communications. They utilized the multi-hop routing protocol that is proposed by $[10,11]$. The maze is a tour puzzle that is composed of roads and walls. From the maze scenario, we describe a storage of knowledge in maze by using a cooperation between SCMs.

Social machine testbed As shown in Figure 7, we set up an SCM in the maze scenario. It is composed of sensors (detecting roads), actuators (DC motor), a battery $(3 \mathrm{~V})$, and a wireless module (IEEE 802.11). In a memory of the machine, it remembers its profile; identification, owner, location information, its neighbors, capabilities, and current interests. By using the wireless module, the machine periodically transmits the machine profile to the other machines.

Maze scenario In Figure 8, we make a description of MSN scenario in the maze, where the social machines try to find a route. There are two social machines in the maze, as shown in Figure 8(a). From broadcasting their profiles, each of machines try to discover the other that has the same interest, the maze. If they find each other, and then they share the map information in Figure 8(b). Here, we use a chat server, in which the SCMs can share their map information with other machines. By using the completed map information, the SCMs find their route in the maze. If one of the SCMs connect to a storage, for example a cloud server, then it sends the information to the storage.

After escaping, another SCM, a machine 3, enters to the maze. As shown in Figure 8(c), the SCM accesses to archive and find the map information that is completed by the machine 1 and 2 . If the machine 3 uses the archive, then it can be more easily find the exit in the maze.

\section{DISCUSSION AND FUTURE WORK}

In this paper, we have suggested the socially connected machines to manage the machines more efficiently. To cope with the scalability problem, we described the characteristics and the requirements capabilities for the socially connected machines. Then, we showed two examples in the machine social networks; the twitbot and the maze machines. As part of the future work, we will set up a testbed for the scenario. We plan to additionally include a socially connect algorithm to connect between follower 


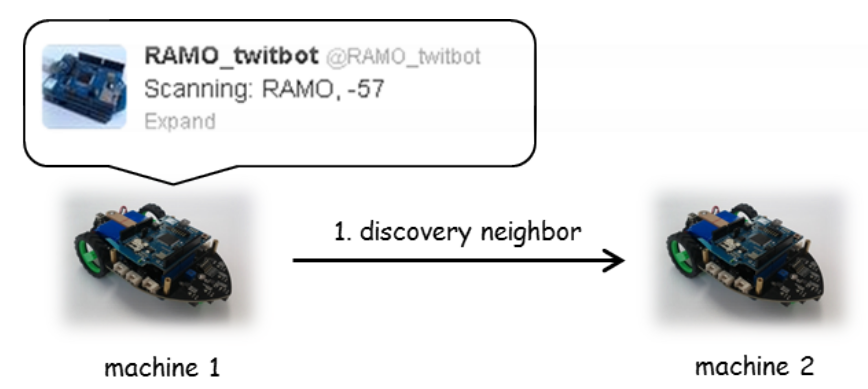

(a) Discovery neighbor.

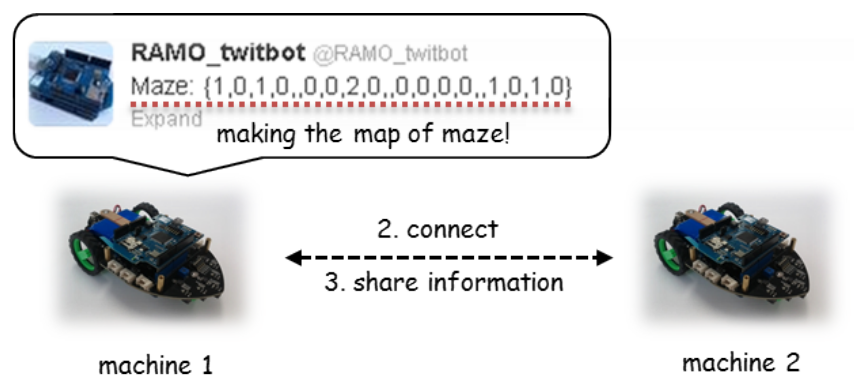

(b) Connect and share informations.

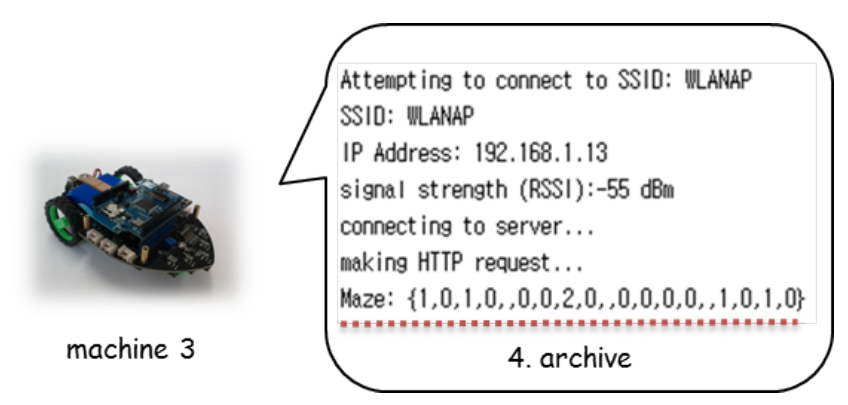

(c) Access to archive.

Fig. 8. Machine social networks scenario in maze.

and followee. Also, we consider that solve a malicious or a broken machine problem.

\section{REFERENCES}

[1] D. Miorandi, S. Sicari, F. D. Pellegrini and I. Chlamtac, Internet of things: Vision, applications and research challenges, Ad Hoc Networks, vol. 10, no. 7, pp. 1497-1516, Sep. 2012.

[2] Ericsson, More than 50 billion connected devices, Ericsson White Paper, Feb. 2011.

[3] L. Atzori, A. Iera and G. Morabito, SIoT: Giving a social structure to the Internet of Things, IEEE Communication Letters, vol. 15, no. 11, pp. 11931195, Nov. 2011.

[4] H. Kwak, C. Lee, H. Park and S. Moon, What is twitter, a social network or a news media?, in Proc. the 19th international conference on World wide web Raleigh, North Carolina, USA, Apr. 2010.

[5] I. Bojic, T. Lipic and V. Podobnik, Bio-inspired clustering and data diffusion in machine social networks, Computational social networks: Mining and visualization / editor: A. Abraham, London, Springer Verlag, pp. 51-79, 2012.

[6] A. Java, X. Song, T. Finin and B. Tseng, Why we twitter: understanding microblogging usage and communities, in Proc. the 9th WebKDD and 1st SNA-KDD 2007 workshop on Web mining and social network analysis, pp. 56-65. Aug. 2007.

[7] M. Kranz, L. Roalter and F. Michahelles, Things that twitter: Social networks and the Internet of Things, Internet of Things do for the Citizen (CIoT) Workshop at The 8th International Conference on Pervasive Computing (Pervasive 2010), Helsinki, Finland, May 2010.

[8] Arduino, http://arduino.cc/.

[9] J. H. Jung, S. Park and S.-L. Kim, Multi-robot path-finding with wireless multihop communications, IEEE Communications Magazine, vol. 48, no. 7, pp. 126-132, Jul. 2010.

[10] D. M. Kim, Y. J. Hwang, S.-L. Kim and G.-J. Jin, Testbed results of an opportunistic routing for multirobot wireless networks, Computer Communications, vol. 34, no. 18, pp. 2174-2183, Dec. 2011.

[11] T. Shim and S.-L. Kim, Robotic Wireless Networks in a Narrow Alley: A Game Theoretic Approach, in Proc. 9th IEEE VTS Asia Pacific Wireless Communications Symposium (APWCS), Kyoto, Japan, Apr. 2012 . 